\title{
BMJ Open Quality Implementation of treatment escalation plans in an old age psychiatry inpatient hospital
}

To cite: Chua AYT, Ghanchi A, Makh SK, et al. Implementation of treatment escalation plans in an old age psychiatry inpatient hospital. BMJ Open Quality 2021;10:e001640. doi:10.1136/ bmjoq-2021-001640

- Additional supplemental material is published online only. To view, please visit the journal online (http://dx.doi.org/10. 1136/bmjoq-2021-001640).

Received 12 August 2021 Accepted 20 November 2021

Check for updates

(C) Author(s) (or their employer(s)) 2021. Re-use permitted under CC BY-NC. No commercial re-use. See rights and permissions. Published by BMJ.

${ }^{1}$ University Hospital Southampton NHS Foundation Trust, Southampton, UK ${ }^{2}$ Southern Health NHS

Foundation Trust, Southampton, UK

${ }^{3}$ University of Southampton, Southampton, UK

Correspondence to

Dr Jay Amin;

jay.amin@soton.ac.uk

\author{
Anne Y T Chua, ${ }^{1}$ Adnaan Ghanchi, ${ }^{1}$ Sangeeta K Makh, ${ }^{1}$ Jessica Grayston, ${ }^{1}$ \\ Stephen J Woolford, ${ }^{1}$ Sahan Wijayaweera, ${ }^{2}$ Victoria Osman-Hicks, ${ }^{2,3}$ \\ Harnish P Patel, ${ }^{1,3}$ Jay Amin (D) ${ }^{2,3}$
}

\section{ABSTRACT}

A treatment escalation plan (TEP) enables timely and appropriate decision making in the management of deteriorating patients. The COVID-19 pandemic precipitated the widespread use of TEPs in acute care settings throughout the National Health Service (NHS) to facilitate safe and effective decision making. TEP proformas have not been developed for the inpatient psychiatric setting. This is particularly concerning in old age psychiatry inpatient wards where patients often have multiple compounding comorbidities and complex decisions regarding capacity are often made. Our aim for this quality improvement project was to pilot a novel TEP proforma within a UK old age psychiatry inpatient hospital. We first adapted a TEP proforma used in our partner acute tertiary hospital and implemented it on our old age psychiatry wards. We then further refined the form and gathered data about uptake, length of time to complete a TEP and the ceiling of care documented in the TEP. We also explored staff, patient and family views on the usefulness of TEP proformas using questionaries. TEP decisions were documented in $54 \%$ of patient records at baseline. Following revision and implementation of a TEP proforma this increased to $100 \%$ on our two wards. The mean time taken to complete a TEP was reduced from 7.1 days to 3.2 days following inclusion of the TEP proforma in admission packs. Feedback from staff showed improvements in understanding about TEP and improved knowledge of where these decisions were documented. We advocate the use of TEP proformas on all old age psychiatry inpatient wards to offer clear guidance to relatives and treating clinicians about the ceilings of care for patients. There are potentially wider benefits to healthcare systems by reducing inappropriate transfers between psychiatry and acute NHS hospitals.

\section{PROBLEN}

Old age psychiatry inpatient wards host older patients who are often living with frailty or have many interacting co-morbidities. These patients are prone to physical decompensation, often requiring medical assessment and intervention. Most old age psychiatry inpatient wards are geographically isolated from acute hospitals and have limited expertise and resources to identify and manage physically deteriorating patients, leading to transfer of patients to local acute hospitals for further assessment and treatment. This is particularly challenging out-of-hours, when transfers of patients with severe mental illness to acute hospitals may occur with limited opportunity to discuss and agree whether this is indeed appropriate. The clinical decision maker can often be a junior doctor on-call or a ward nurse who may not know the patient care plan and family's prior wishes. This can lead to inappropriate invasive procedures or distressing episodes of care, which could have otherwise been avoided with clear advanced care planning.

Despite their widespread use in acute hospitals, a formal treatment escalation plan (TEP) is rarely used in inpatient old age psychiatry hospitals. This project took place at the Western Community Hospital (WCH), Southern Health National Health Service (NHS) Foundation Trust, Southampton. WCH hosts two old age psychiatry inpatient wards. Berrywood ward accommodates 18 older adults with functional mental illnesses such as schizophrenia and depression. Beaulieu ward accommodates up to 14 older adults with severe behavioural symptoms of dementia. The two wards are staffed by a multidisciplinary team (MDT) including two consultant psychiatrists, junior doctors, clinical psychologists, occupational therapists, physiotherapists and a team of nurses and healthcare support workers. The two wards serve a large geographical area across the central South Coast, with the hospital situated one mile away from the local acute tertiary hospital, University Hospital Southampton NHS Foundation Trust (UHSFT).

The project team, which included specialists in old age psychiatry and medicine for older people, were thrust into making timely decisions about the upper limit of care that should be provided for our inpatients in the event of clinical deterioration during the first 
wave of the COVID-19 pandemic in early 2020. Prior to this, discussions about these upper limits of care, termed 'ceiling of care', were not routine and were inconsistently documented, with feedback from on-call doctors highlighting this issue. We believed that clearer documentation of these ceiling of care discussions could be greatly improved for the benefit of our patient group, and that implementation of TEP forms would be the best way to achieve this.

Our aim was to develop a TEP proforma that would be implemented in at least $80 \%$ of patients on our old age psychiatry inpatient wards.

\section{BACKGROUND}

TEPs offer greater clarity in planning appropriate decision making in the management of deteriorating patients by explicitly stating a ceiling of care, where these decisions are discussed with and agreed by patients or relatives. ${ }^{1}$ This includes, but is not limited to, a Do Not Attempt Cardiopulmonary Resuscitation (DNACPR) decision, as well as decisions regarding organ failure support (eg, non-invasive ventilation) and appropriate settings of care for deteriorating patients (eg, whether intensive care unit admission would be suitable). Completion and regular review of a TEP ensures that patients are not subject to inappropriate invasive procedures or distressing modes of care in the case of terminal events. However, a TEP also ensures that all necessary and suitable treatment is started for a patient who is deteriorating, aiding the decision making of clinicians, especially on-call junior clinicians, who have not been previously involved in that patient's care during their admission.

The use of TEP proformas have been adopted in several acute hospitals across the NHS with success, ${ }^{2} 3$ with one previous quality improvement project showing the highest usage rates on acute wards for older people, demonstrating the importance of TEP in patients who may be more frail. ${ }^{4}$ Another project demonstrated that $96 \%$ of sampled patients and relatives thought that a TEP would be beneficial. ${ }^{1}$ A further project noted that DNACPR forms were not sufficient to guide clinical decisions about treatment escalation and showed that TEP proformas not only benefited the patient but also the treating clinicians, particularly on-call junior doctors. ${ }^{5} \mathrm{~A}$ retrospective case note review of 45 cases concluded that formal TEP proformas were needed to enable visible and clear management plans for deteriorating patients, especially due to the complex management of such patients. ${ }^{6}$ One previous quality improvement project found the introduction of standardised TEP proformas significantly improved the clarity of documented treatment decisions, ${ }^{7}$ and another showed that on-call doctors found documented ceiling of treatment proformas highly beneficial. ${ }^{8}$ Lastly, there are challenges in the completion of a TEP but methods, such as use of an information leaflet, can improve the proportion of inpatients who had a TEP. ${ }^{9}$
The COVID-19 pandemic increased awareness of the importance of discussions regarding DNACPR and ceiling of care due to the risk of rapid deterioration in patients with COVID-19 and limited bed space in hospitals, especially in settings providing respiratory organ support. The pandemic provided an opportunity to strengthen clinical decision making about treatment escalation. ${ }^{10}$ National Institute for Health and Care Excellence (NICE) COVID-19 rapid guidelines recommended timely discussion of ceiling of care decisions with patients and families, and documentation of these decisions through a TEP. ${ }^{11}$ Although the COVID-19 pandemic accentuated the need for TEPs, these should be adopted as part of good clinical practice, especially in older people who may be living with frailty.

Prior to the COVID-19 pandemic there was no TEP proforma in use at WCH. Discussions about ceilings of care were not consistently documented on the electronic patient record system ( $\mathrm{RiO}$ ), leading to some uncertainty about whether treatment escalation was appropriate. This was particularly problematic when the treating clinician had no prior knowledge of the patient, such as when out-of-hours doctors were called about a deteriorating patient. Therefore, the design and implementation of a TEP proforma was identified to be an area that we could improve patient care on the old age psychiatry inpatient wards at the WCH.

\section{BASELINE MEASUREMENT}

Baseline data were collected cross-sectionally from electronic patient records of all inpatients with ongoing admissions on 30 July 2020 across both Berrywood and Beaulieu wards at WCH. Each patient's notes were scrutinised for documentation of any type of discussion about ceiling of care with the patient or their family. This excluded discussions solely about DNACPR.

Of the 24 patients present in hospital at baseline assessment, $13(54 \%)$ patient records had evidence of a ceiling of care discussion being documented with either the patient themselves or their family. However, there was often some ambiguity about the final ceiling of care decision and precisely who was consulted.

Baseline qualitative data were collected through a staff questionnaire exploring views about ceiling of care and TEP (online supplemental figure 1). Staff members were chosen based on stratified sampling that consisted of three junior doctors, two consultants, one healthcare assistant and four nurses.

We planned to collect data again following the design and implementation of the TEP proforma at WCH.

\section{DESIGN}

In order to meet our project aim of designing a TEP proforma for the old age psychiatry inpatient hospital, we modified the TEP proforma already in use at our neighbouring acute hospital (UHSFT) after permission was 
granted. This previous proforma included the following levels of care:

- Level 3=consider critical care (multi-organ support, advanced respiratory support).

- Level 2=critical care step down.

- Level 1=patients monitored on observable bay with critical care outreach team input.

- Level $0=$ active ward-based care.

The project team discussed how this TEP proforma could be adapted to suit an old age psychiatry community inpatient hospital. With input from the multidisciplinary team (MDT) the following list described the level of wardbased intervention available for inpatients at WCH: oral medication, subcutaneous fluids, bloods tests, radiological investigations (facilitating appointments at UHSFT), physical observation monitoring and electrocardiogram. Any higher level of care, such as intravenous medication or critical care, would necessitate transfer to UHSFT. An option of palliative care was also deemed to be necessary. Following agreement within the project team an adapted TEP proforma was implemented on both wards at WCH. We anticipated some anxiety among staff about using a new proforma, and of the implications of a TEP recommending that a patient not be transferred to the acute hospital. We therefore provided education sessions for the wider multidisciplinary team about the TEP proforma, the rationale for its implementation and provided time for any questions to be answered. Following rollout of the TEP we intended to review the proforma to see if any further improvements could be made.

\section{STRATEGY}

\section{Plan-Do-Study-Act (PDSA) cycle 1}

In our first PDSA quality improvement cycle, we implemented the adapted TEP proforma on both old age psychiatry inpatient wards at WCH in August 2020. The senior and junior medical team prompted ceiling of care discussions with patients and family members (where appropriate) for existing inpatients and all new admissions. Feedback about the TEP proforma led to several minor changes, with the final version shown in figure 1 .

First, a requirement to include the patient's address was removed since two points of identification were already in place (name and date of birth or NHS number). A requirement was added to state the person or persons with which the ceiling of care discussion was had. We believed this to be good practice, enabling re-discussion with the same person should the TEP need to be reviewed. We changed the heading 'for ward-based care' to make it explicitly clear that this was for care on the psychiatry ward and not ward-based care at the acute hospital. Lastly, to aid clarity we changed the DNACPR section from a free text line to a tick box.

Data on whether a TEP had been completed, the outcome of the TEP, who contributed to the discussion and the number of days from admission it took to complete the TEP was collected on 25 September
Treatment Escalation Plan (TEP)

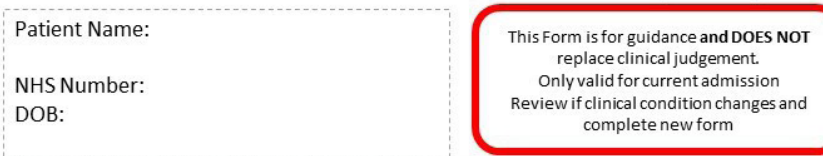

Has a discussion of TEP with the patient/relatives occurred? Yes $\square \quad$ No $\square$ If no, why?

Name of person discussed with

Relation to patient

For Full Escalation to Tertiary Care

For Western $\mathrm{CH}$ Ward Based Care:

Oral antibiotics

Subcutaneous Fluids

Yes $\square \quad$ No $\square$

Blood Tests

Radiological Investigations

ECG

Regular Medications

Yes $\square \quad$ No $\square$

Yes $\square \quad$ No $\square$

Yes $\square \quad$ No $\square$

Yes $\square \quad$ No $\square$

Yes $\square \quad$ No $\square$

For Palliative Care

DNACPR status

DNACPR $\square \quad$ FOR RESUS $\square$

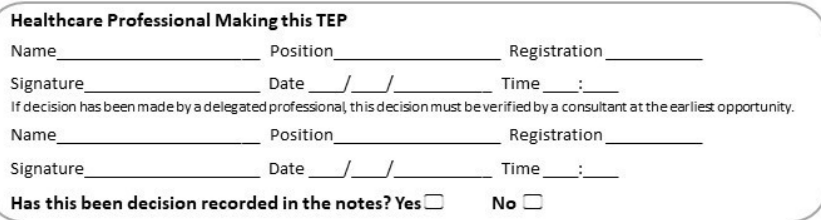

Figure 1 TEP proforma. DANCPR, Do Not Attempt Cardiopulmonary Resuscitation; NHS, National Health Service; Resus, Resuscitation

2020 retrospectively by reviewing electronic and paper records for all admissions since baseline $(n=20)$. TEP proforma completion had improved to $100 \%$ from $54 \%$ at baseline, with the mean duration from admission to proforma completion lasting 7.1 days.

After implementation of the TEP proforma, a staff questionnaire was again used to explore views about the TEP proforma, including staff confidence about comprehensiveness of the proforma and whether staff felt that decisions were easily locatable (online supplemental figure 1). Again, a stratified sampling method was adopted to include seven junior doctors, three consultants, three MDT members (including occupational therapist, physiotherapist and psychologist) and seven nurses.

\section{PDSA cycle 2}

The widespread use of the TEP proforma provided benefit to regular and on-call staff in delivering more clarity on decisions related to ceiling of care. However, patients had often been admitted for over a week before completion of the TEP proforma. We, therefore, included a blank TEP proforma within the admission clerking proforma that the inpatient junior medical 


\begin{tabular}{llll}
\hline Table 1 & Number of inpatients with a TEP & \\
\hline & Baseline & $\begin{array}{l}\text { PDSA } \\
\text { cycle 1 }\end{array}$ & $\begin{array}{l}\text { PDSA } \\
\text { cycle 2 }\end{array}$ \\
\hline $\begin{array}{l}\text { Number of inpatients } \\
\text { Number of inpatients }\end{array}$ & 24 & 20 & 21 \\
with a TEP proforma & $13(54 \%)$ & $20(100 \%)$ & $21(100 \%)$ \\
\hline
\end{tabular}

PDSA, Plan-Do-Study-Act; TEP, treatment escalation plan.

team used when new patient admissions were assessed. This prompted early discussions with patients and/or family about ceiling of care and meant that the TEP proforma could often be finalised by a senior doctor before the initial Care Planning Approach meeting on the ward.

Further data was gathered on 3 November 2020 and showed that TEP proforma completion was maintained at $100 \%$ of all admissions $(n=21)$, with the mean duration from admission to proforma completion reduced to 3.2 days from 7.1 days.

Patients and their relatives were approached by telephone $(n=10)$ to complete a questionnaire (online supplemental figure 2) to gather their views on whether they understood what a TEP form was and if they felt it would be useful to have one in place.

\section{RESULTS}

Our primary outcome measure was the number of inpatients who had a TEP proforma. The completion of a TEP proforma increased from $54 \%$ at baseline to $100 \%$ following PDSA cycle 1 and was maintained at $100 \%$ following PDSA cycle 2 (table 1 ).

The mean time to complete a TEP proforma from admission reduced from 7.1 days to 3.2 days in PDSA cycle 2 (table 2), likely due to the inclusion of the TEP proforma in admission clerking packs. In both PDSA cycles the majority of TEP decisions were made following consultation with a family member rather than the patient themselves. Unexpectedly, in both PDSA cycles most TEP proformas supported full escalation to the acute hospital.

\begin{tabular}{|c|c|c|}
\hline & PDSA cycle 1 & PDSA cycle 2 \\
\hline Number of inpatients & 20 & 21 \\
\hline $\begin{array}{l}\text { Time from admission } \\
\text { to completion of TEP } \\
\text { (mean days) for new } \\
\text { admissions }\end{array}$ & 7.1 & 3.2 \\
\hline $\begin{array}{l}\text { Who was consulted } \\
\text { in decision making } \\
\text { process }\end{array}$ & $\begin{array}{l}2 \text { with patient } \\
17 \text { with family } \\
1 \text { neither }\end{array}$ & $\begin{array}{l}3 \text { with patient } \\
17 \text { with family } \\
1 \text { neither }\end{array}$ \\
\hline $\begin{array}{l}\text { Ceiling of care stated } \\
\text { in TEP }\end{array}$ & $\begin{array}{l}18 \text { full escalation } \\
2 \text { ward-based } \\
\text { care } \\
0 \text { palliative care }\end{array}$ & $\begin{array}{l}19 \text { full escalation } \\
2 \text { ward-based care } \\
0 \text { palliative care }\end{array}$ \\
\hline
\end{tabular}

PDSA, Plan-Do-Study-Act; TEP, treatment escalation plan.
A questionnaire was distributed to different members of the MDT before PDSA cycle 1 and after the TEP proforma had been implemented. Staff reporting of understanding what a TEP is, comprehensiveness of TEP proformas and ease of location of a TEP decision were dramatically improved. A further questionnaire was distributed to patients/family members after PDSA cycle 2 . This showed that the understanding of what a TEP was and the desire to have a TEP in place was very high among both patients and their family members.

\section{LESSONS AND LIMITATIONS}

Introducing a TEP proforma into patient admission clerking proformas drastically improved uptake of TEP decision making, with likely benefits to patients' safety and care within our cohort. Simply using the same TEP proforma as an acute general hospital was not adequate to meet the needs of an old age psychiatry inpatient hospital. Adaption was required to allow for the differences in availability of medical interventions, but this was relatively straightforward to accommodate.

Although the COVID-19 pandemic was the precipitant for this project, the importance of having a TEP in old age psychiatry is clear. This is especially so on organic wards that host people with severe dementia, where escalation of treatment to an acute general hospital may not always be appropriate. One noteworthy study provided data regarding advance care planning of patients with severe dementia in a nursing home setting and reported on implementation of a 'Goals of Care' intervention, consisting of a video decision aid and structured care planning meeting for family decision makers, in a cluster randomised trial. The intervention was shown to be effective at enhancing palliative care plans and reducing transfers to acute hospitals. ${ }^{12}$ Discussions about ceiling of care should form part of standard clinical practice in any setting hosting people with severe dementia, including on old age psychiatry inpatient wards.

Our project revealed that most discussions when agreeing to a TEP were had with family members of patients rather than solely with patients themselves. This is likely to have been because many of the patients on the organic dementia ward, and indeed the functional ward, did not have mental capacity to make decisions regarding their TEP. Although many patients were still consulted for their views even if they lacked capacity, some patients with severe dementia were not able to express a preference. The COVID-19 pandemic generated significant anxiety in family members of patients, exacerbated by their inability to visit their loved ones. The discussion about an appropriate ceiling of care is a difficult one, further complicated by the need to limit these discussions to telephone or video calls only. The use of information leaflets for patients and relatives, as has previously been tested, ${ }^{9}$ may help to prime for these discussions. Furthermore, it is important to remember that TEP decisions are not final and they should be periodically reviewed with 
patients and/or families, particularly if there are significant changes to the patient's medical status.

One important limitation to this project was the lack of exploration of the downstream effects of having more TEPs in place. This study did not collect data on the number of transfers to the acute hospital, and whether these were appropriate. Analysis of cost savings of reduced inappropriate transfers would have been a powerful argument to support use of TEP. Additionally, feedback from out-of-hours medical and nursing staff on the accessibility and clarity of the TEP proforma would have allowed further refinement.

An unexpected finding from this project was the relatively high proportion of patients whose TEP supported full escalation to the acute general hospital. Although some of these patients did not have multiple comorbidities and were physically well aside from their mental health disorder, on reflection, a significant proportion of the patients probably would not have benefited from escalation of treatment. There are several reasons why our project observed such a high proportion of TEPs that supported escalation of care to the acute hospital. First, the decision to advocate for escalation of treatment may have been influenced by anxiety in the nursing and allied healthcare team, who may not have felt they had sufficient skills and confidence in managing deteriorating patients, including those at end-of-life. Second, the largely remote mode of discussion during COVID-19 pandemic restrictions is likely to have affected the quality of discussion about benefits and drawbacks of escalation. Lastly, more junior medical staff may not have been confident enough, or had the necessary support, to undertake these discussions.

The disproportionate number of patients who were for escalation is an important area that warrants further review. One possible area for improvement is to ensure adequate training for junior and senior doctors in psychiatry having discussions about ceiling of care, to ensure their practice is in line with local geriatricians. This may be possible through sessional general practitioner or geriatrician input into the wards. It may be that junior psychiatry doctors require a greater consultant presence to facilitate and answer questions about TEP with patients and families. In addition, good clinical practice dictates an early senior review for new admissions, allowing an opportunity for a consultant to lead on these discussions soon after admission. It has been shown previously that large numbers of hospital inpatients enter the last year of their lives following admission. ${ }^{13}$ This may be even less adequately recognised in psychiatry settings, impacting on escalations of treatment being too high in comparison to when patients with dementia are admitted to acute hospitals.

In the era of electronic patient records, it could be argued that the use of paper TEP proformas alone is outdated. The implementation of TEP decisions on electronic patient records has been demonstrated in the NHS and is a development that we would support. ${ }^{14}$ This would improve the accessibility of TEP proformas, especially important in psychiatry where on-call doctors are nonresident and therefore out-of-hours staff may not have immediate access to paper records.

Our TEP proforma was designed to be tailored to the range and nature of treatment available at the WCH. Therefore, if other settings are able to provide a different level of care, such as intravenous fluids, our TEP proforma would need to be adapted to each specific care setting. If our patients are transferred to an acute general hospital then a new TEP proforma is completed, one that contains options including ward-based care versus escalation to intensive care. Similarly, if our patients are discharged from hospital to a nursing home then an adapted TEP proforma could be designed to complement locally used advance care planning documentation.

\section{CONCLUSION}

TEPs play an important role in guiding clinical decision making. Our project demonstrates that TEP proformas can be successfully adapted and implemented on two old age psychiatry inpatient wards. We exceeded our aim of having $80 \%$ of patients having a TEP proforma in place, and in fact achieved a completion rate of $100 \%$. We thereby significantly reduced ambiguity regarding ceiling of care decisions for our patient group. We did, however, find that an unexpectedly high number of patients, including those with severe dementia, had a TEP that advocated transfer to the acute hospital. We conclude that while it is important to have a TEP in place, there must be sufficient training and support for those undertaking these discussions to enable the most appropriate ceiling of care to be agreed.

We acknowledge that there is a pressing need for clarity on treatment escalation decisions across old age psychiatry inpatient units, with no previous quality improvement project published in this field. The prevention of unnecessary admissions to acute general hospitals is likely to provide both a cost saving to the local healthcare economy but also enable the most appropriate care to be delivered to patients. However, to support old age psychiatry wards to manage patients who are not for escalation, we postulate that enhanced training and staffing is required to support the medical assessment and management of these patients. Future projects could include analysis of cost savings of preventing inappropriate transfers to acute hospitals along with the impact of this on medical assessment and care on the old age psychiatry wards.

The project team have begun to support other inpatient wards in our healthcare organisation in rolling out our TEP proforma, with further refinement in the discussion and documentation process likely to be beneficial. We now aim to have our TEP proforma adopted onto our electronic patient record system and accompanied by guidance on its use, which will improve the sustainability of our intervention. Widespread adoption of the 
TEP proforma will allow continuation of the work started by the project team, enabling treating clinicians to access clear guidance about the ceiling of care for older mental health inpatients.

Acknowledgements We thank the patients, families and staff who participated in this quality improvement project.

Contributors JA and HPP conceived the study. AYTC, AG, SKM, JG, SJW and JA contributed to data collection, analysis and interpretation. AYTC, AG, SKM, JG, SJW, SW, VO-H, HPP and JA drafted, revised and approved the manuscript for submission. JA was responsible for the overall content as guarantor.

Funding The authors have not declared a specific grant for this research from any funding agency in the public, commercial or not-for-profit sectors.

Competing interests None declared.

Patient consent for publication Not required.

Ethics approval This was a quality improvement project that did not require ethics approval.

Provenance and peer review Not commissioned; externally peer reviewed.

Data availability statement Data are available upon request.

Supplemental material This content has been supplied by the author(s). It has not been vetted by BMJ Publishing Group Limited (BMJ) and may not have been peer-reviewed. Any opinions or recommendations discussed are solely those of the author(s) and are not endorsed by BMJ. BMJ disclaims all liability and responsibility arising from any reliance placed on the content. Where the content includes any translated material, BMJ does not warrant the accuracy and reliability of the translations (including but not limited to local regulations, clinical guidelines, terminology, drug names and drug dosages), and is not responsible for any error and/or omissions arising from translation and adaptation or otherwise.

Open access This is an open access article distributed in accordance with the Creative Commons Attribution Non Commercial (CC BY-NC 4.0) license, which permits others to distribute, remix, adapt, build upon this work non-commercially, and license their derivative works on different terms, provided the original work is properly cited, appropriate credit is given, any changes made indicated, and the use is non-commercial. See: http://creativecommons.org/licenses/by-nc/4.0/.

ORCID iD

Jay Amin http://orcid.org/0000-0003-3792-0428

\section{REFERENCES}

1 Obolensky L, Clark T, Matthew G, et al. A patient and relative centred evaluation of treatment escalation plans: a replacement for the donot-resuscitate process. J Med Ethics 2010;36:518-20.

2 Paes P, O'Neill C. Treatment escalation plans - a tool to aid end of life decision making? BMJ Support Palliat Care 2012;2:A60.1-A60.

3 Dartford \& Gravesham NHS Trust. Reducing mortality through improving care for 'acutely ill patients in hospital', 2013.

4 Stockdale C, Trivedi B, Jerome E, et al. Implementation of a combined cardiopulmonary resuscitation and treatment escalation plan document in a district general Hospital. BMJ Qual Improv Rep 2014;2. doi:10.1136/bmjquality.u202653.w1236. [Epub ahead of print: 09 Dec 2013].

5 Shermon E, Munglani L, Oram S, et al. Reducing DNACPR complaints to zero: designing and implementing a treatment escalation plan using quality improvement methodology. BMJ Open Qual 2017;6:e000011.

6 Campling N, Cummings A, Myall M, et al. Escalation-related decision making in acute deterioration: a retrospective case note review. BMJ Open 2018;8:e022021.

7 Zainal T, Healy D, Joyce C, et al. 176New Proforma for DNAR and treatment escalation plan (TEP) improves quality of documentation in a large teaching hospital. Age Ageing 2018;47:v1-12.

8 Dahill M, Powter L, Garland L, et al. Improving documentation of treatment escalation decisions in acute care. BMJ Qual Improv Rep 2013;2:u200617.w1077-1077.

9 Sayma M, Nowell G, O'Connor A, et al. Improving the use of treatment escalation plans: a quality-improvement study. Postgrad Med J 2018;94:404-10.

10 McIntosh L. Can the COVID-19 crisis strengthen our treatment escalation planning and resuscitation decision making? Age Ageing 2020;49:525

11 National Institute for Health and Care Excellence. COVID-19 rapid guideline: managing COVID-19, 2021.

12 Hanson LC, Zimmerman S, Song M-K, et al. Effect of the goals of care intervention for advanced dementia: a randomized clinical trial. JAMA Intern Med 2017;177:24-31.

13 Clark D, Armstrong M, Allan A, et al. Imminence of death among hospital inpatients: prevalent cohort study. Palliat Med 2014;28:474-9.

14 Johnson M, Whyte M, Loveridge R, et al. A unified electronic tool for CPR and emergency treatment escalation plans improves communication and early collaborative decision making for acute hospital admissions. BMJ Qual Improv Rep 2017;6:u213254. w6626. 